\title{
Analysis Combustion Gas Composition of Solid Waste
}

\author{
Li-Hua Song ${ }^{a, *}$ \\ Department of Chemistry Engineering, Huainan Union University, Huainan, 232001 \\ alihuasong2000@126.com
}

Keywords: Combustion, Solid waste, Analysis

\begin{abstract}
Analyzed, the results show that the EGA analysis can effectively characterize solid waste in each stage of pyrolysis. Using the EGA technology, such as paint. RDF release in different stages of the gas composition in the combustion process.Gasification and combustion dynamic characteristics. To master the research of waste incineration process mechanism, composition and the corresponding influencing factors, the corresponding model is established.
\end{abstract}

\section{Introduction}

Waste is car paint, chemical and other industries of industrial waste, such as cannot carry on the effective treatment can cause resource waste and environmental pollution. To study and analysis in the laboratory using the instrument, and then applying research results to industrial equipment, has the advantages of high efficiency, low investment. Sludge mainly includes make water to the harm of environmental deterioration of water quality, pollution of soil and crops, etc.[1]Traditional methods of sludge treatment and disposal (landfill) because of the high cost, and easy to cause secondary pollution, incineration is feasible processing method. Good combustion organization is the first condition of efficient and clean combustion, the combustion conditions can reduce pollutants through reasonable control original generation.[2]

\section{Equipment}

Experiments using thermo gravimetric analyzer is Germany NETZSCH STA409 thermogravimetric analyzer. Fourier transform infrared spectroscopy (FTIR, the core equipment for the Michael interferometer. Due to the synchronous measurement requirements and thermogravimetric analyzer, so is preheated to a certain stage in thermogravimetric analyzer, infrared analyzer should also enter a state of preheating, set the preheating temperature of the airway (set to $200^{\circ} \mathrm{C}$ ).

\section{Experiment and Discussion}

For some typical waste (paint, sludge, garbage derivatives, etc.) the pyrolysis - oxidation test, the data is obtained. FTIR sampling parameters in experiments were not change, always be: resolution, $4 \mathrm{~cm}^{-1}$; Scanning way, double-sided bidirectional; Scanning speed, $40 \mathrm{kHz}$. The Times of scanning and 16 times.

The IR spectrum of SPPO, CLSPPO, triazole- and tetrazole-membranes are showed in Figure.1. The spectra for SPPO (a) is characterized by a band at $1192 \mathrm{~cm}^{-1}$ corresponding to the $-\mathrm{S}=\mathrm{O}$ bond, and those corresponding to $-\mathrm{SO}_{3}{ }^{-}$groups are observed at 1026 and $1066 \mathrm{~cm}^{-1}$. After chlorosulfonation of the SPPO with thionyl chloride, a new band corresponding to $-\mathrm{SO}_{2} \mathrm{Cl}$ group appeare at $1374 \mathrm{~cm}^{-1}$ on the spectra for CISPPO (b) and then the $-\mathrm{SO}_{3}{ }^{-}$groups disappeared. This observation is agree with previous IR studies on chlorosulfonated membranes.[3] After reaction with $-\mathrm{NH}_{2}$ of the MOFs or NAPI, the $-\mathrm{SO}_{2} \mathrm{Cl}$ band totally disappeare and a new band at $1170 \mathrm{~cm}^{-1}$, corresponding to $-\mathrm{SO}_{2} \mathrm{NH}-$ group, confirm the acid-amine bonding in Figure (c) and (d).[4] 


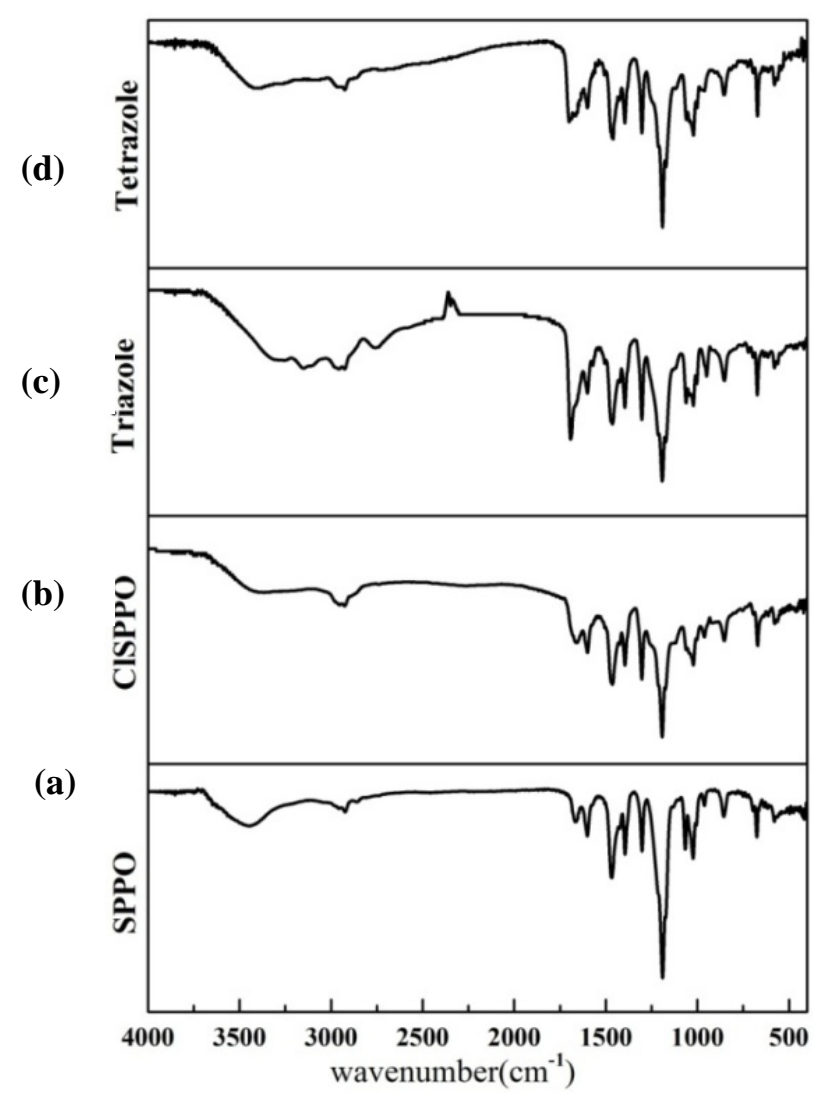

Fig.1. IR spectrum of (a) SPPO, (b)ClSPPO , (c)and (d)triazole- and tetrazole-membranes

[1]HNMR spectroscopy is suitable for charactering the capability of target molecules reactivity and adsorptivity.[5]We therefore use this analytical technique to determine the sulfonylation reaction with CISPPO and amino in Heterocycle. The [1]HNMR spectrum (DMSO) of the triazoleand tetrazole-membranes are shown in Figure. 2. We can observe that one signal at $\delta_{\mathbf{H}}=7.4 \mathrm{ppm}$ caused by the sulfamide groups in membrane triazole (a) and tetrazole (b).[6]

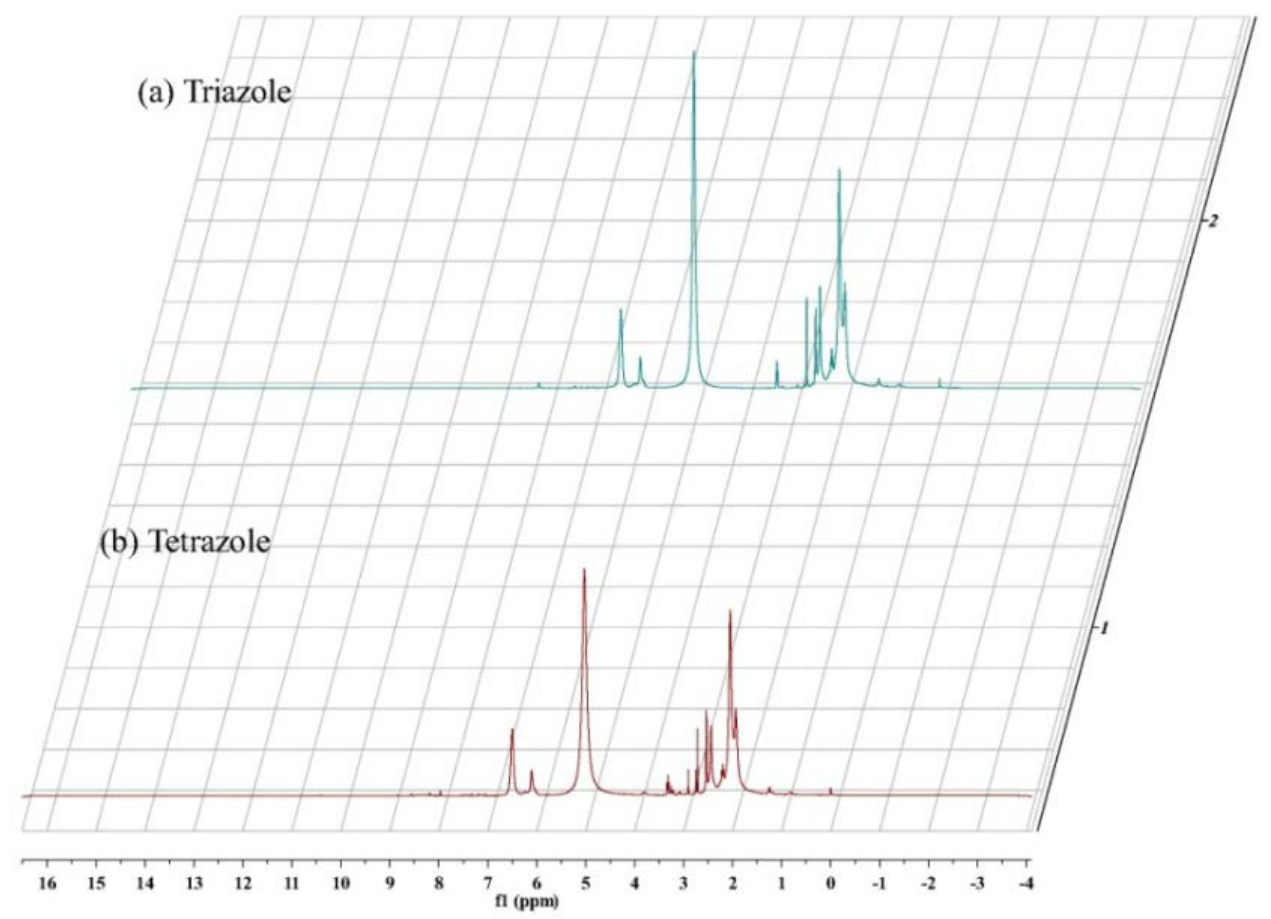

Fig. $2 .{ }^{1} \mathrm{H}$ NMR spectra of thetriazole- and tetrazole-membranes. 
A typical membrane is chosen for conductivity measurements. Figure. 4 shows the results as temperature-dependent values $\left(\sigma\right.$ vs $\mathrm{T}^{-\mathbf{1}}$ ) for both the heating and cooling regimes. The proton conductivities of membrane at low temperatures and ambient humidity (relative humidity $(\mathrm{RH})=$ $25 \%$ ) is $2.5 \times 10^{-4} \mathrm{~S} \cdot \mathrm{cm}^{-1}$. Such values match well to the conductivities of the corresponding liquid heterocycle taking into account the partial filling of the pores and its low conducting properties.[7]Increasing the temperature resulted in a gradual increase in the proton conductivity, although the slope of the curve decreased at the same time. Such a concave profile of the temperature- dependent conductivity curves for the as-synthesized membrane upon heating can be very well explained by the proton transfer of the heterocycle.

The second cycle of heating-cooling experiments shows a similar trend as the first one, and then the deviations between the heating and cooling profiles are less pronounced (Figure 3). Most importantly, the cooling lines for the two experiments almost coincided, indicating that the proton conductivity properties of the membrane follow a predictable pattern once the adsorbance of hetercycle is stabilized at higher temperatures.

The discrepancies between the heating and cooling regimes are due to the kinetics of the hetercycle adsorption. The frameworks structures of them are maintained constant over the whole temperature range, the proton generation and conductivity of the membrane would likely follow the curve trend. Another important conclusion is that any temperature changes along with the corresponding alterations of the ambient humidity have less effect on the hetercycle content. [8] In other words, the durability of the membrane is not that low, as could be expected from the hetercycle-impregnated porous structures. The hetercycle molecules play an important role in increasing the proton concentration by loading the hetercycle as well as by expanding the proton-conducting pathways through the hydrogen bonding network, which eventually enhance the mobility of the protons.[9]

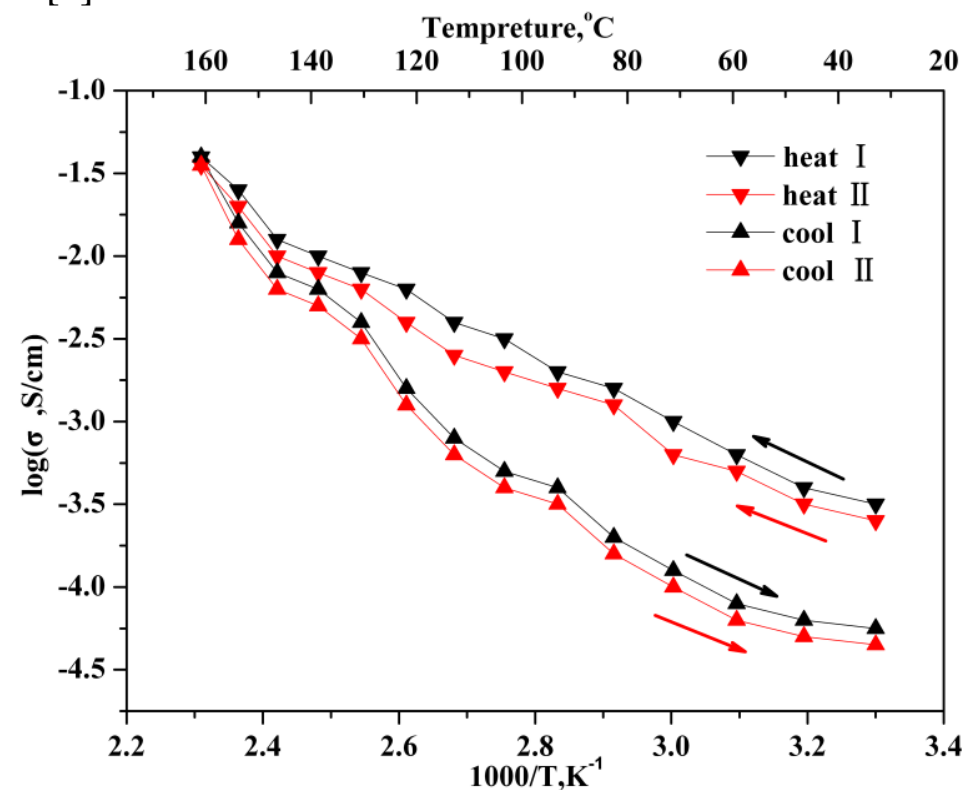

Fig.3. Temperature dependence of the proton conductivities of membrane in heating-cooling cycles.

Black and red triangles indicate twice processes of heating (heat) and cooling (cool) regimes, respectively.

The proton conductivity in membrane arise from the contribution of two pathways. (i) Structure diffusion (Grotthuss-type mechanism): the protons are transferred between fixed, adjacent binding sites through intermolecular proton transfer within hydrogen bonded network or structure followed by hydrogen bond breaking and formation.[10]In this case, the conductivity of stationary protons and hetercycle intermolecular mainly depends on structure diffusion. (ii) vehicular diffusion (vehicle-type mechanism): the protons are bound to and carried by mobile species and conduct via a long range diffusion of the protonated carrier molecules. In our case, the adsorbed hetercycle carry protons to mobile for long distance conduction by this approach. This research suggests that the 
hetercycle is the main reason for enhanced proton conductivity in the membrane under high temperature.[11]

\section{Conclusions}

We put forward a novel approach to prepare PEMs based on the use of proton-carrier organic molecules to enhance the proton conductivity at high temperature under anhydrous condition. Here, we selected hetercycle as proton-carrier organic molecules which are absorbed into MOFs first, then suffer the sulfone amidation reaction with CISPPO to obtain the PEMs. The proton conductivities at $160^{\circ} \mathrm{C}$ is $0.04 \mathrm{~S} / \mathrm{cm}$, which surpasses the pure MOFs-based PEM and meanwhile can be comparable to those polymeric PEMs. The proton conductivity is promoted by the protons from the sulfonimide in polymeric chain and hetercycle itself, and the hetercycle molecules guarantee continuous proton conduction. For better understanding, we propose a new possible pathway of the proton transfer to show that the Grotthuss-type mechanism and vehicle-type mechanism work simultaneously in the membrane.

\section{References}

[1] S. J.Paddison, Annu. Rev. Mater. Res. 33, 289, (2003).

[2] M. F. H.Schuster, W. H. Meyer,M.Schuster, K. D. Kreuer, Chem. Mater. 16, 329, (2004).

[3] M.Winter, R. J. Brodd, Chem. Rev. 104 , 4245, (2004).

[4] A.Noda, M.Susan, K.Kudo, S.Mitsushima,K.Hayamizu, M. Watanabe, J. Phys. Chem. B. 107, 4024, (2003).

[5] C.Yang, P.Costamagna, S.Srinivasan, J.Benziger, A. B. Bocarsly, J. Power Sources 103, 1, (2001).

[6] W.Deng, V.Molinero, W. A.Goddard, J. Am. Chem. Soc. 126, 15644, (2004).

[7] T.Sata, R.Izuo, K. Takata, J. Membr. Sci. 451, 97, (1989).

[8] S.Horike, R. Matsuda, M. Takata, Chem. Commun. 9, 2152, (2004).

[9] T.Ueda, K. Kurokawa, T. Eguchi, Chem. Phys. Lett. 443, 293, (2007).

[10] G. Alberti, M.Casciola, Solid State Ionics.145, 3, (2001).

[11] T. J.Peckham, S.Holdcroft, Adv. Mater. 22, 4667, (2010). 